\title{
土壤养分斑块对比度改变活血丹克隆整合强度和方向
}

\author{
张丽丽 ${ }^{1,2}$ 董 鸣 $^{1}$ 李仁强 ${ }^{1,2}$ 王艳红 ${ }^{1,2}$ 崔清国 ${ }^{1,2}$ 何维明 $1^{*}$ \\ (1 中国科学院植物研究所植被与环境变化国家重点实验室 北京 100093) \\ (2 中国科学院研究生院 北京 100049)
}

摘 要 克隆植物相连片段 (或分株) 常常生长在不同的土壤养分斑块中。克隆整合使得生长在异质养分斑块中 的克隆片段(或分株)产生局部和非局部反应，从而影响相连片段(或分株)的表型可塑性。为了揭示养分斑块对比 度对活血丹 ( Glechoma longituba) 克隆整合的影响，在一控制实验中 将活血丹克隆片断种植于 4 种不同对比度的环 境中，即 无对比度(对照)、低对比度、中对比度和高对比度。活血丹在气体交换、水势、苂光、形态、生长与分配方 面的克隆整合强度随养分斑块对比度的增强而表现出增强或减弱的变化趋势; 养分斑块对比度越强, 活血丹气体 交换和苂光暗反应的整合强度越小，叶片水势整合强度越大。斑块对比度可改变部分性状的克隆整合方向; 超过 一定的对比國值, 整合强度随养分对比度的变化趋势会向着相反方向转变。克隆整合对生理特征的修饰幅度小于 对生长特征的修饰幅度。这些结果指示: 养分斑块对比度可通过修饰克隆整合格局(即强度和方向)而改变克隆植 物的表型可塑性。

关键词 克隆可塑性 气体交换 生理整合 生长与分配 养分斑块 苂光特征

\section{SOIL-NUTRIENT PATCH CONTRAST MODIFIES INTENSITY AND DIRECTION OF CLONAL INTEGRATION IN GLECHOMA LONGITUBA}

\author{
ZHANG Li-Li ${ }^{1.2}$, DONG Ming ${ }^{1}$, LI Ren-Qiang ${ }^{12}$, WANG Yan-Hong ${ }^{12}$, CUI Qing-Guo ${ }^{12}$, and HE Wei- \\ Ming $^{1 *}$ \\ ${ }^{1}$ Key Laboratory of Vegetation and Environmental Change , Institute of Botany , Chinese Academy of Sciences , Beijing 100093 , China , and \\ ${ }^{2}$ Graduate University of Chinese Academy of Sciences , Beijing 100049 , China
}

\begin{abstract}
Aims The connected fragments of clonal plants often grow in different soil-nutrient patches , and clonal integration can modify the plasticity of these connected clonal fragments in diverse ways. We address how nutrient patch contrast modifies intensity and direction of clonal integration.

Methods In a common garden experiment, we planted clonal fragments of Glechoma longituba in four nutrient-patch contrast conditions : no (control), low, medium and high contrast. Water was supplied when necessary. We measured physiological traits at peak growth and harvested all plant materials at the end of the experiment, which ran from July 19 through September 30 , 2005. Data were analyzed with SPSS software.

Important findings Clonal integration in gas exchange, water potential , fluorescence, growth and biomass allocation differed among nutrient patch contrast treatments. Higher contrast had smaller integration in both gas exchange and dark fluorescence response and greater integration in leaf water potential. Direction of clonal integration for some characteristics also different among treatments, indicating that the threshold of soil-nutrient patch contrast might play a fundamental role in determining integration direction. Clonal integration had far broader effects on growth and allocation than physiological traits. We infer that nutrient-patch contrast may modify the intensity and direction of clonal integration, which in turn can shape the phenotypic plasticity of clonal plants.
\end{abstract}

Key words clonal plasticity , fluorescence, gas exchange, growth and allocation , nutrient patches , physiological integration 
间上突出表现为斑块状分布 (Svesson \& Callaghan, 1988 ; 董鸣, 1996 ; Farley \& Fitter , 1999)。克隆植物 的不同分株或片段常常生长在不同的土壤养分斑块 中 (Hutchings \& de Kroon 1994; Farley \& Fitter , 1999)。克隆分株间在形体上的相连使生长在异质 环境中的分株间能够实现物质转移，从而使分株间 产生克隆生理整合 (Hutchings \& de Kroon 1994 ; 董 鸣, 1996)。克隆整合使得处于某一小生境中的分 株既可对所处小生境发生局部反应, 也可对与其相 连的分株所处的小生境发生非局部反应 (Turkington et al ., 1991 ; Dong，1995；Alpert，1999)。克隆整合 帮助克隆植物有效地利用异质性资源, 提高资源利 用率 增加种群适合度(张称意等 2001)。

斑块对比度, 即相邻斑块中资源水平的对比度, 是斑块性的基本要素(Stuefer, 1996)。养分斑块所 决定的土壤养分空间分布及其可利用性与根系吸收 能力共同制约着叶片养分含量, 而后者又与叶片的 气体交换密切相关(何维明和董鸣,2002)。克隆植 物具有鲜明的等级性结构，如枝叶等构件、分株、克 隆片段和基株。以前的研究表明 斑块对比度能改 变克隆植物不同等级水平的反应特征 (何维明和董 鸣 2002)。克隆整合修饰产生的结果可能是正的也 可能是负的，从而使局部反应增强或减弱 (Evans， 1992 ; Dong，1995)。本文以克隆植物活血丹( Glechoma longituba) 为对象, 探讨土壤养分斑块对比度 对克隆植物在生理和生长方面整合强度和方向的影 响。作者假设 :1)在一定的斑块对比范围内, 克隆整 合强度随养分斑块对比度的增强而增强, 因为这种 变化有利于克隆植物适应斑块状分布的自然资源;

2) 克隆整合方向可能随养分斑块对比度发生变化, 因为这有利于提高克隆植物适应变化环境的灵活 性 3)在相同的对比梯度下, 整合作用对生理特征 (快反应) 的修饰作用比它对生长特征 (慢反应) 的修 饰作用更强烈，因为生理反应和生长反应的时间尺 度存在差异。

\section{1 材料和方法}

\section{1 研究物种}

活血丹系唇形科活血丹属, 多年生克隆草本, 具 细长匍匐茎, 逐节着地生根。除青海、甘肃、新疆及 西藏外, 全国各地均有分布; 常常生长在海拔 50 $2000 \mathrm{~m}$ 范围内的林缘、疏林下、草地中、溪边等阴湿 生境中。该植物通过产生较长的地上匍匐茎表现出 克隆生长习性。实验材料于 2005 年 3 月采自北京
东灵山自然生长种群。

\section{2 实验设计}

该实验于 2005 年 7 月 19 日至 9 月 30 日在中国 科学院植物研究所进行。7 月初移栽实验材料于装 有细沙的不透水槽内 $(1.2 \mathrm{~m} \times 0.2 \mathrm{~m} \times 0.3 \mathrm{~m})$, 两 个克隆片断中间用挡板隔开防止养分和水分交换， 只有间隔子相连，使生长在不同养分斑块中的同一 克隆植株的两个片段能够进行物质交换 (图 1)。整 个槽底到槽外沿覆有塑料薄膜防止养分及水分流 失。

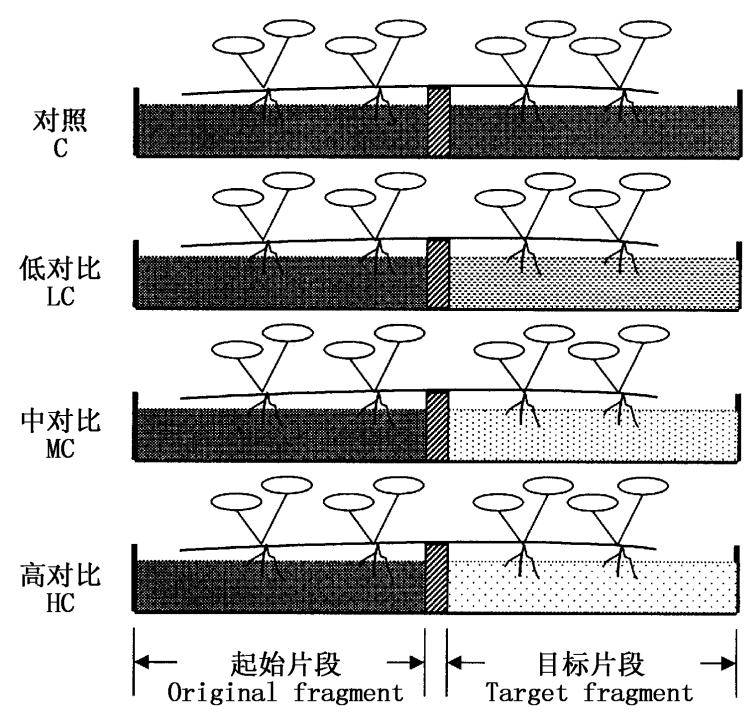

图 1 实验设计示意图 (生长槽中点的浓淡深浅表征养分浓度的高低)

Fig. 1 Layout of the experiment (The four different patterns stand for four different nutrient patches)

C : Control LC : Low contrast MC : Medium contrast HC : high contrast

经过预实验, 施肥浓度太高会引起烧苗, 浓度太 低则不同处理间可能没有显著差异。因此, 施肥浓 度在 $0.05 \% \sim 0.50 \%$ 之间比较合适。实验处理包括 4 个养分对比强度, 即对照 (起始斑块和目标斑块的 施肥浓度均为 $0.45 \%$ 总养分浓度为 $1125 \mathrm{~g} \mathrm{~m}^{-3}$ )， 低对比 (起始斑块施肥浓度为 $0.45 \%$,总养分浓度 为 $1125 \mathrm{~g} \mathrm{~m}^{-3}$; 目标斑块施肥浓度为 $0.4 \%$ 总养分 浓度为 $1000 \mathrm{~g} \mathrm{~m}^{-3}$ ), 中对比 (起始斑块施肥浓度为 $0.45 \%$,总养分浓度为 $1125 \mathrm{~g} \mathrm{~m}^{-3}$; 目标斑块施肥 浓度为 $0.35 \%$,总养分浓度为 $875 \mathrm{~g} \mathrm{~m}^{-3}$ ), 高对比 (起始斑块施肥浓度为 $0.45 \%$,总养分浓度为 1125 $\mathrm{g} \mathrm{m}^{-3}$; 目标斑块施肥浓度为 $0.3 \%$,总养分浓度为 $750 \mathrm{~g} \mathrm{~m}^{-3}$ )(表 1)。因此, 中对比处理的强度为低对 比处理的两倍, 而高对比处理的强度则为低对比 的3 倍。所用肥料为 Scotts Peters Professional 花多多 
表 1 不同对比养分斑块中的养分状况

Table 1 Total amount of nutrients used in the four contrasting nutrient patches

\begin{tabular}{|c|c|c|c|c|c|c|c|c|}
\hline & \multicolumn{8}{|c|}{ 养分总量 Total amount of nutrients $\left(\mathrm{g} \mathrm{m}^{-3}\right)$} \\
\hline & \multicolumn{2}{|c|}{$\begin{array}{c}\text { 对照 } \\
\text { Control (C) }\end{array}$} & \multicolumn{2}{|c|}{$\begin{array}{c}\text { 低对比强度 } \\
\text { Low contrast (LC) }\end{array}$} & \multicolumn{2}{|c|}{$\begin{array}{c}\text { 中对比强度 } \\
\text { Medium contrast (MC) }\end{array}$} & \multicolumn{2}{|c|}{$\begin{array}{c}\text { 高对比强度 } \\
\text { High contrast (HC) }\end{array}$} \\
\hline & O & $\mathrm{T}$ & O & $\mathrm{T}$ & 0 & $\mathrm{~T}$ & $\mathrm{O}$ & $\mathrm{T}$ \\
\hline $\mathrm{N}$ & 1125 & 1125 & 1125 & 1000 & 1125 & 875 & 1125 & 750 \\
\hline $\mathrm{P}$ & 1125 & 1125 & 1125 & 1000 & 1125 & 875 & 1125 & 750 \\
\hline K & 1125 & 1125 & 1125 & 1000 & 1125 & 875 & 1125 & 750 \\
\hline
\end{tabular}

$\mathrm{O}$ :初始片段 Original fragment $\mathrm{T}$ :目标片段 Target fragment

20-20-20 型，N、P、K 含量均为 $20 \%$ 。实验选用大小 相似的两个相连分株, 新产生的分株在槽内生根, 并 经历与母株相同的生长条件。每个处理每周施相应 浓度的营养液, 适时适量施水使水分条件正常满足 植物生长。每一处理设 6 个重复。

\section{3 数据收集和统计分析}

9 月 14 日，用 LI-6400 便携式光合仪测定气体 交换 ,9月 25 日，用叶绿素苂光仪(PAM-101/102/103 Chlorophyll Fluorescence System, Heinz Walz GmbH, Germany)测叶片的苂光反应; 9 月 28 日，用水势仪

( PSYPRO Water Potential System, Wescor, Inc. , USA) 测植物叶片的水势。生理指标测定分别在初始片段 和目标片段上进行 ,3 4 个重复。10 月 3 日,分别 收获初始片段和目标片段, 将每个片段连根挖出, 洗 净, 分拣成叶片、叶柄、间隔子和根 4 部分; 从每个片 段中随机选取 3 个叶片、叶柄、间隔子和根, 并测定 叶面积、叶柄长、间隔子长度和根长。将所有样品放 在 $80{ }^{\circ} \mathrm{C}$ 干燥箱中烘至恒重, 用万分之一天平分别称 叶片、叶柄、间隔子和根生物量。为了量化整合强度 指标,作者提出一个计算公式 :

整合强度 $I=\Sigma 2 \times\left(X_{o j}-X_{t j}\right) /\left(X_{o j}+X_{t j}\right)$

式中 : $X_{o j}$ 表示初始片段的第 $j$ 个特征值, 而 $X_{t j}$ 则表 示目标片段的第 $j$ 个特征值。 $I$ 值越大, 整合强度越 小。

本实验为单因素四水平设计, 养分斑块对比度 为固定因子, 用 SPSS 软件中 One-Way ANOVA 方法 分析实验处理对整合强度的效应。

\section{2 结 果}

活血丹叶片生理指标的克隆整合强度对养分斑 块对比度的反应存在明显差异 (图 2)。气体交换特 征(净光合速率、气孔导度、胞间环境浓度比和蒸腾 速率)的整合强度, 随养分对比的增大而呈减小趋 势;所有目标片段的气体交换速率均大于初始片段 (图 2A)。叶片水势整合强度随养分斑块对比的增
大而增强; 在低对比和中对比条件下,初始片段的水 势低于目标片段, 在高对比条件下恰好相反 (图 $2 \mathrm{~B}$ )。不同苂光特征 (最小苂光、最大苂光、最大光 化学量子产量、光化学淬灭系数和非光化学淬灭系 数)的整合强度随养分斑块对比的变化趋势存在差 异。就光反应而言, 斑块生境中的最大苂光整合强 度大于同质生境, 最大苂光整合强度随斑块对比的 增强而减小;所有斑块生境中目标片段的最大苂光 都大于起始片段 (图 2C)。对照生境和低斑块对比 生境中的最大光量子产量和光化学淬灭系数整合强 度高于中对比和高对比养分斑块生境; 中对比养分 条件下,目标片段最大光化学量子产量和光化学淬 灭系数小于起始片段，其余对比强度下恰好相反(图 $2 \mathrm{C}$ )。最小荧光整合格局与最大苂光恰好相反 (图 $2 \mathrm{C}$ )。在苂光暗反应中, 斑块环境中目标片段的最 大光化学量子产量小于起始片段, 而最小苂光和最 大苂光正好相反; 最大苂光整合强度随斑块对比的 增大而增大, 而最大光化学量子产量呈相反的变化 趋势(图 2D)。

在低对比和中对比条件下,分株片断生物量的 整合强度不受斑块对比的影响; 但高斑块对比显著 影响克隆整合，即目标片段的叶生物量小于起始片 段 (图 3A)。叶生物量分配的整合强度随斑块对比 的增大呈波形变化; 中对比条件下，目标片段的叶生 物量分配大于起始片段, 其余对比条件下相反 (图 $3 \mathrm{~A})$ 。中斑块对比中茎生物量分配小于低和高斑块 对比; 在低对比条件和高对比条件下, 目标片段的生 物量大于起始片段(图 3A)。叶柄生物量分配、根生 物量分配和根冠比的整合强度, 均在对照和低斑块 对比中较大，而在中和高斑块对比中较小; 所有斑块 对比中，起始片段的叶柄生物量均大于目标片段; 除 低对比条件外，其余斑块对比条件下的起始片段根 生物量比和根冠比均大于目标片段 (图 3A)。比生 物量整合强度均随斑块对比的增大呈 波浪” 型变 化，低斑块对比中的初始片段与目标片段最相似; 在 

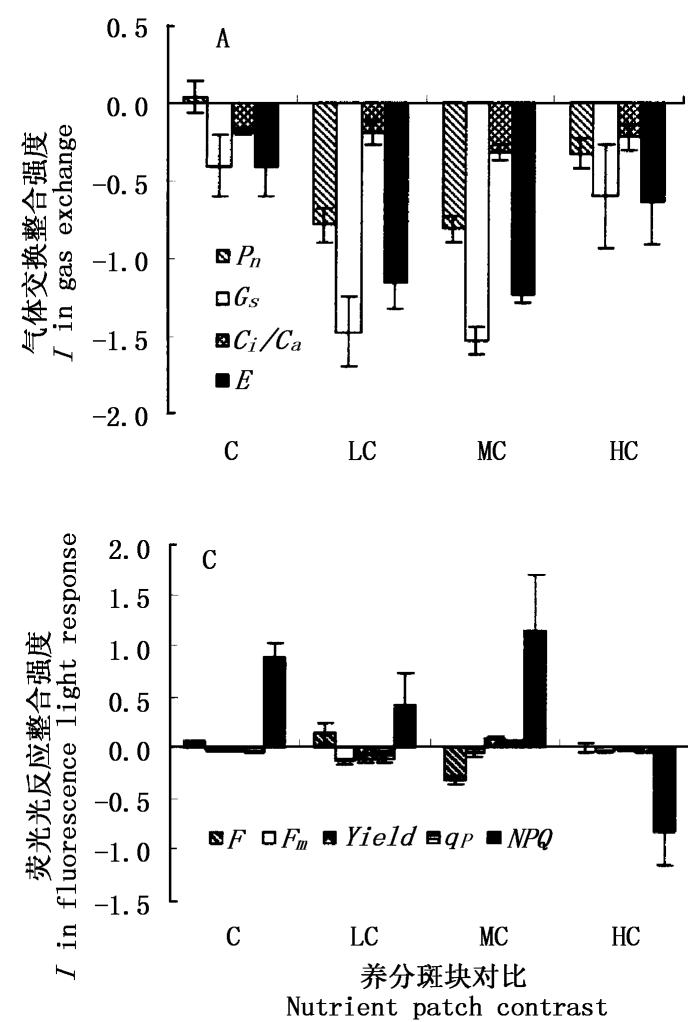
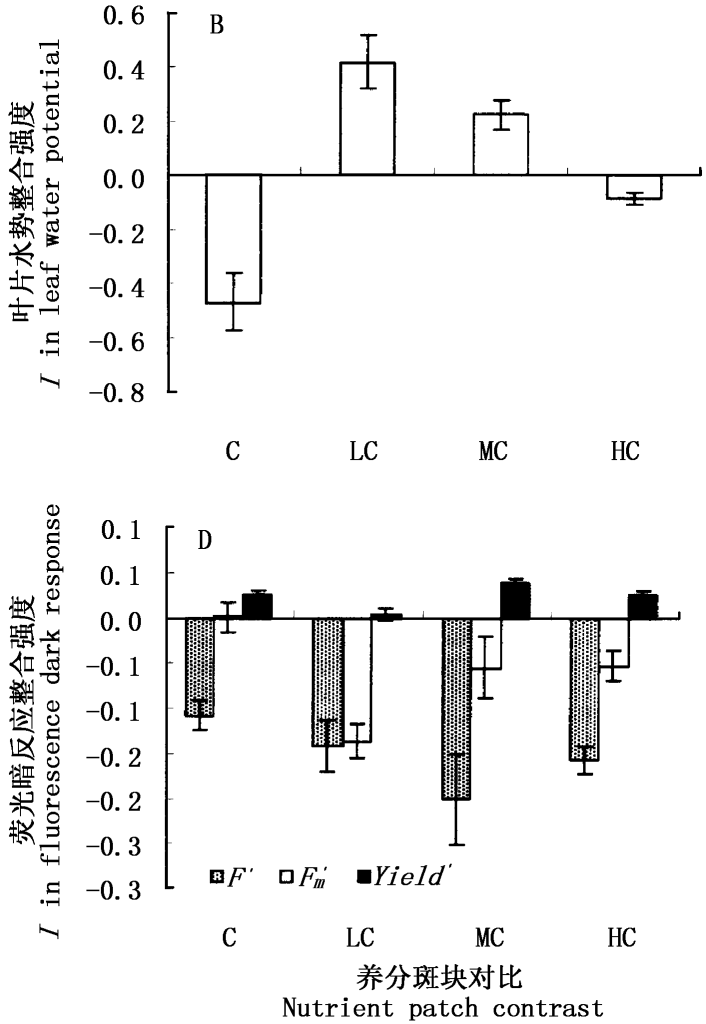

Nutrient patch contrast

图 2 不同养分斑块对比度下气体交换 (A) 水势 (B)和苂光反应 $(\mathrm{C}, \mathrm{D})$ 的克隆整合格局

Fig. 2 Clonal integration $(I)$ in physiological function along a nutrient-patch contrast gradient

$P_{n}$ 旪片净光合速率 Net photosynthetic rate $G_{s}$ :气孔导度 Stomatal conductance $C_{i} / C_{a}$ :胞内外 $\mathrm{CO}_{2}$ 浓度比 Ratio of substomatal $\mathrm{CO}_{2}$ to atmospheric $\mathrm{CO}_{2} E$ 蒸腾速率 Transpiration rate $F$ 最小苂光 Minimal fluorescence in the light conditions $F_{m}$ :最大苂光 Maximal fluorescence in the light conditions Yield 最大光化学量子产量 Fluorescence efficiency of PS II in the light conditions $q_{P}$ 光化学淬灭系数 Photochemical quenching in the light conditions $N P Q$ 非光化学淬灭系数 Nonphotochemical quenching in the light conditions $F^{\prime}$ 最小苂光 Minimal fluorescence in the dark conditions $F_{m}{ }^{\prime}$ : 最大菼光 Maximal fluorescence in the dark conditions Yield' 最大光化学量子产量 Fluorescence efficiency of PS II in the dark conditions C、LC、MC、HC : 同图 1 See Fig. 1

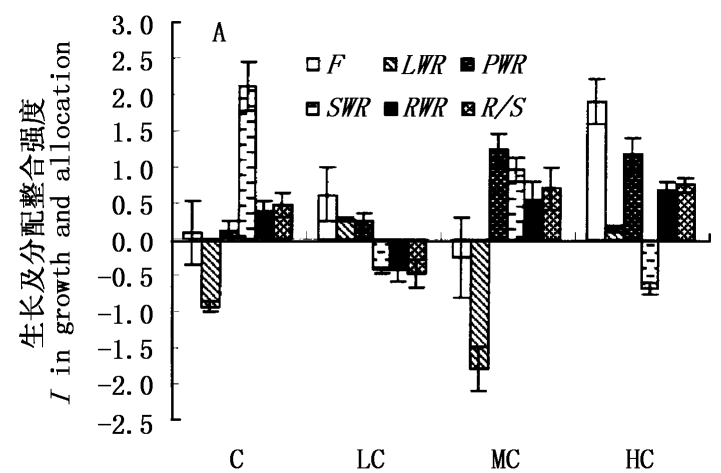

养分斑块对比

Nutrient patch contrast

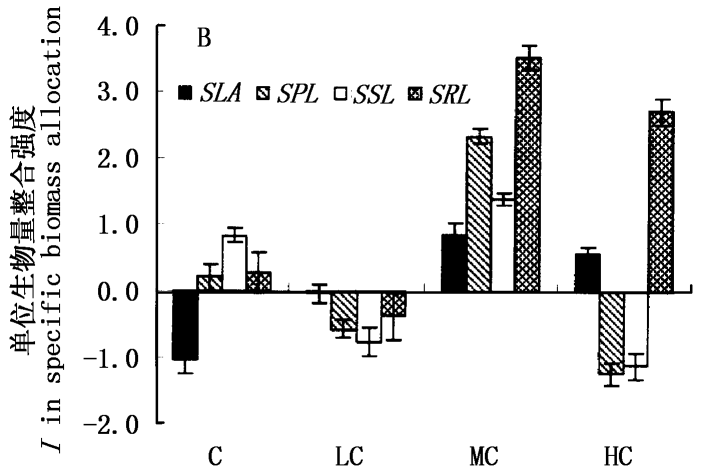

养分斑块对比

Nutrient patch contrast

图 3 养分斑块对比度对生长与分配 (A)和比生物量分配 (B)整合强度的影响

Fig.3 Clonal integration (I) in biomass growth and allocation (A) and specific biomass allocation (B) along a nutrient-patch contrast gradient

$F$ :克隆片段生物量 Fragment biomass LWR 旪片生物量比 Lamina weight ratio PWR 叶柄生物量比 Petiole weight ratio SWR :匍匐茎生 物量比 Stolon weight ratio RWR 根生物量比 Root weight ratio $R / S$ 根冠比 Root/shoot ratio $S L A$ : 比叶面积 Specific lamina area $S P L$ : 比叶柄长 Specific petiole length $\quad S S L$ : 比茎长 Specific stolon length SRL :比根长 Specific root length $\quad$ C、LC、MC、HC: 同图 1 See Fig. 1 
恰好相反(图 3B)。

\section{3 讨 论}

克隆植物对斑块性状 (如大小、尺度)的表型反 应已经引起人们的极大兴趣, 但对斑块性本身的研 究却非常有限 (Hodge, 2004)。理论研究表明:生理 整合可能在高斑块对比情况下有所加强 (Eriksson \& Jerling ,1990； Caraco \& Kelly ,1991)。但支持或反对 这一假说的实验证据还很少 ( Stuefer \& Hutchings , 1994 ; Wijesinghe \& Handel ,1994)。生理整合程度与 环境斑块性程度和环境中平均资源有效性有关;在 资源匮乏但未受干扰的环境中, 生理整合程度高 (Caraco \& Kelly , 1991 ; Stuefer et al. , 1994 ; Hutchings \& Wijesinghe , 1997 ; Jónsdóttir \& Watson , 1997)。 在养分斑块对比环境中，气体交换特征(净光合速 率、胞间/环境 $\mathrm{CO}_{2}$ 浓度比、气孔导度和蒸腾速率等) 的整合强度随斑块对比强度的增强而减小; 叶片水 势整合强度随斑块对比强度的增强而增大 ;苂光反 应,尤其是光反应中, 克隆整合随斑块对比强度的增 强而增大 就生长和生物量分配而言, 克隆整合也随 养分斑块对比的增强而呈现增大趋势。因此,这些 结果表明 活血丹的多数性状的克隆整合随养分斑 块对比的增强而呈现增大趋势, 也有部分性状的克 隆整合随养分斑块对比的增强而呈现减小趋势。所 以，引言中的第一假设被部分支持。植物不同组织 水平对资源差异响应的时间尺度不同, 使其对相同 外界环境刺激的反应结果也存在差异。不同组织水 平上的克隆内资源转移和形态可塑性可能表征潜在 的适应特征, 使得克隆植物能更好地适应斑块性生 境(Stuefer, 1996)。活血丹的生理整合强度随斑块 对比度的变化格局表明 随着养分对比的增强, 起始 片段放弃一部分自身生长和生理的需要, 资助处于 相对低养分斑块中的与其相连的目标片段, 以使其 适应相对不良的环境。通过耗费一定的资源以满足 分株间的克隆整合这一过程使得基株的适合度从中 获得净收益(张称意等,2001)。

生理整合是一个相连克隆分株间实现物质转移 的过程 (Hutchings \& de Kroon, 1994 ; 董鸣, 1996)。 作者提出的克隆整合强度公式不仅能反映克隆整合 强度, 还可以反映克隆整合的 净方向”。克隆整合 指数的正负表明 (图 2 , 图 3 ) :资源转移随养分斑块 对比的增强/减弱而增强/减弱到一定程度就改变方 向 或者称其为 波浪型” 变化。这一结果支持了引 言中的第二个假设，即整合的方向不是固定的，会随
着资源源-汇关系的不同而改变。在大多数克隆植 物中, 资源的向顶转移 (比如老株到幼株) 占绝对优 势, 而母株与分株间的互惠资源转移通常很有限 (Pitelka \& Ashmun，1985）。然而，也有研究报道了资 源的向基转移。这种整合被认为是一种对生长季中 一种或多种资源低于平均可利用性的适应机制 (Jónsdóttir \& Watson , 1997)。似乎通过提供保存贵 乏资源的方法, 促进分株间的发育分工, 开拓复合生 境中斑块性分布的资源, 使处于资源匮乏环境中的 植物获利，并提供一种机制以加强对基部分株的克 隆内调控 (Jónsdóttir \& Watson，1997)。随着斑块对 比强度增强到一定程度(养分中到高对比之间)整合 强度反而减弱, 可能是由于养分对比强度过大 (例 如, 高养分对比条件下, 目标分株所处的斑块资源相 对匮乏)使得成本-收益平衡被打破，以至于目标片 段所获得的收益不足以补偿进行克隆整合后起始片 段的损失 植株减弱或拒绝克隆整合。

生理过程常常是快过程，因为其周转时间较短; 相反, 生长过程是慢过程, 因为其周转时间相对较 长。尽管生理性状普遍表现出整合性, 但其变化范 围相对较小（图 2); 生长和分配指标中有些性状并 没有表现出强烈的整合性, 但其变化的范围较大(图 3)。因此,这些结果不能支持引言中提出的第三个 假设，即相同的对比梯度下，整合作用对生理特征的 修饰作用比它对生长特征的修饰作用更强烈。生长 和形态特征是植物对外界环境适应性的外在表现， 生理反应是植物对变化的外界环境的最直接的反 应。生长和形态指标是从整体上把握生理指标反映 的结果，生理指标则是更灵敏更深刻地反映植物对 环境变化的响应机制的一种表征。由于生理和生长 特征处于不同的时间尺度,有时形态上未表现出对 环境变化的响应, 往往其生理指标已经发生了变化。

斑块对比是推动整合作用的一种主要外动力 (Stuefer，1996），使得资源在克隆内进行转移。斑块 对比使相连分株间建立了源-汇关系。有时, 克隆内 的资源转移并非由环境斑块性造成, 而是克隆内不 同源 (如成年克隆构件) 和汇(如建立的分株)间发育 的不同引起的 (Jónsdóttir \& Callaghan，1990)。低养 分斑块中的目标分株由于得到与其相连的起始分株 的资助, 通过产生相对大和厚的叶片以及更长的叶 柄以期获得更多的光合产物, 适应相对较低的养分 供应和不利的生境。活血丹植株内进行了克隆整 合, 使得某一克隆分株不仅对其所处的小生境条件 发生局部反应, 而且也可对与其相连的克隆构件所 
处的小生境条件发生非局部反应，这两种反应共同 影响克隆植物的生长发育和繁殖等生理活动, 进而 影响植株的适合度 (Evans，1992)。高养分小生境更 趋向于产生高密度的植被 (Stuefer \& Hutchings, 1994)。环境中资源丰富 (土壤养分) ,植株将更多的 资源投资给地上部分，此时个体间地上部分的竞争 (尤其是光竞争) 成为主要的生长限制因素 (单保庆 等 2000)。比叶柄长在低和高养分对比斑块中均表 现出较强的克隆整合性。这表明植株可能通过增长 源分株叶柄的长度或增大叶面积和叶的厚度以求获 取更多的光资源，产生更多光合产物。比间隔子长 是克隆植物选择性地将克隆分株或片段放置在合适 斑块中的重要手段, 高对比斑块中的匍匐茎生物量 分配和单位长度匍匐茎生物量都表现出显著的克隆 整合性, 可能表示植株试图通过增加对目标分株匍 匐茎的投资,使其尽快脱离不利的生存环境。

\section{参考文 献}

Alpert P (1999). Effects of clonal integration on plant plasticity in Fragaria chiloensis. Plant Ecology, 141, 99-106.

Caraco T, Kelly CK (1991). On the adaptive value of physiological integration in clonal plants. Ecology, 72, 81-93.

Dong M (1995). Morphological responses to local light conditions in clonal herbs from contrasting habitats, and their modification due to physiological integration. Oecologia, 101, 282-288.

Dong M (董鸣) (1996). Clonal growth in plants in relation to resource heterogeneity: foraging behavior. Acta Botanica Sinica (植 物学报), 88, 828-835. (in Chinese with English abstract)

Eriksson O, Jerling L (1990). Hierarchical selection and risk spreading in clonal plants. In: van Groenendael J, de Kroon $\mathrm{H}$ eds. Clonal Growth in Plants: Regulation and Function. SPB Academic Publishing, the Hague, the Netherlands, $79-94$.

Evans JP (1992). The effect of local resource availability and clonal integration on ramet functional morphology in Hydrocotyle bonariensis. Oecologia, 86, 268-275.

Farley RA, Fitter AH (1999). The response of seven co-occurring woodland herbaceous perennials to localized nutrient-rich patches. Journal of Ecology, 87, 849-859.

He WM (何维明), Dong M (董鸣) (2002). Ramets and genets in the tillering clonal herb Panicum miliaceum in hierarchical response to heterogeneous nutrient environments. Acta Ecologica Sinica (生态学报), 20,169-175. (in Chinese with English abstract)
Hodge A (2004). The plastic plant: root responses to heterogeneous supplies of nutrients. New Phytologist, 162, 9-24.

Hutchings MJ, de Kroon H (1994). Foraging in plants: the role of morphological plasticity in resource acquisition. Advances in Ecological Research, 25, $159-238$.

Hutchings MJ, Wijesinghe DK (1997). Patchy habitats, division of labour and growth dividends in clonal plants. Trends in Ecology and Evolution, 12, $390-394$.

Jónsdóttir IS, Callaghan TV (1990) . Intraclonal traslocation of ammonium and nitrate in Carex bigelowii using ${ }^{15} \mathrm{~N}$ and nitrate reductase assays. New Phytologist, 114, 419 - 426.

Jónsdóttir IS, Watson MA (1997). Extensive physiological integration: an adaptive trait in resource-poor environments? In: de Kroon H, van Groenendael J eds. The Ecology and Evolution of Clonal Plants. Backhuys Publishers, Leiden, the Netherlands, $109-136$.

Pitelka LF, Ashmun JW (1985) . Physiology and integration of ramets in clonal plants. In: Jackson JBC, Buss LW, Cook RE eds. Population Biology and Evolution of Clonal Organisms. Yale University Press, New Haven, London, $1-55$.

Shan BQ (单保庆), Du GZ (杜国祯), Liu ZH (刘振恒) (2000). Clonal growth of Ligularia virgaurea: morphological responses to nutritional variation. Acta Phytoecologica Sinica (植物 生态学报), 24, 46-51. (in Chinese with English abstract)

Stuefer JF, During HJ, de Kroon H (1994). High benefits of clonal integration in two stoloniferous species, in response to heterogeneous light environments. Journal of Ecology, 82, 511- 518 .

Stuefer JF, Hutchings MJ (1994) . Environmental heterogeneity and clonal growth: a study of the capacity for reciprocal translocation in Glechoma hederacea L. Oecologia, 100, 302-308.

Stuefer JF (1996) . Potential and limitations of current concepts regarding the response of clonal plants to environmental heterogeneity. Vegetatio, 127, 55-70.

Svesson BM, Callaghan TV (1988). Small-scale vegetation pattern related to the growth of Lycopodium annotinum and variations in its micro-environment. Vegetatio, 76, $167-177$.

Turkington R, Hamilton RS, Gliddon C (1991). Within population variation in localized and integrated responses of Trifolicum repens to biologically patchy environments. Oecologia, 86, 183-192.

Wijesinghe DK, Handel SN (1994). Advantages of clonal growth in heterogeneous habitats: an experiment with Potentilla simplex. Journal of Ecology, 82, 495 - 502.

Zhang CY (张称意), Yang C (杨持), Dong M (董鸣) (2001). The clonal integraion of photosynthate in the rhizomatous halfshrub Hedysarum leave. Acta Ecologica Sinica (生态学报), 21, 1986 - 1993. (in Chinese with English abstract) 